OPEN ACCESS

Edited by:

Carlo Sala

Italian National Research Council, Italy

Reviewed by:

Andreas Martin Grabrucker

University of Limerick, Ireland

Hans-Jürgen Kreienkamp,

University Medical Center

Hamburg-Eppendorf, Germany

${ }^{*}$ Correspondence:

Kihoon Han

neurohan@korea.ac.kr

tThese authors have contributed equally to this work

Received: 06 October 2020 Accepted: 02 December 2020 Published: 11 January 2021

Citation:

Jin C, Kang H, Yoo T, Ryu JR, Yoo $Y$-E, Ma R, Zhang Y, Kang HR, Kim Y, Seong H, Bang G, Park S, Kwon S-K, Sun W, Kim H, Kim JY,

Kim E and Han K (2021) The Neomycin Resistance Cassette in the Targeted Allele of Shank3B

Knock-Out Mice Has Potential Off-Target Effects to Produce an

Unusual Shank3 Isoform Front. Mol. Neurosci. 13:614435 doi: 10.3389/fnmol.2020.614435

\section{The Neomycin Resistance Cassette in the Targeted Allele of Shank3B Knock-Out Mice Has Potential Off-Target Effects to Produce an Unusual Shank3 Isoform}

\author{
Chunmei Jin $1,2 t$, Hyojin Kang ${ }^{3 t}$, Taesun Yoo ${ }^{4 t}$, Jae Ryun Ryu ${ }^{5}$, Ye-Eun Yoo, \\ Ruiying Ma ${ }^{1,2}$, Yinhua Zhang 1,2, Hyae Rim Kang ${ }^{1,2}$, Yoonhee Kim ${ }^{1}$, Hyunyoung Seong ${ }^{1}$, \\ Geul Bang ${ }^{7,8}$, Sangwoo Park ${ }^{7}$, Seok-Kyu Kwon ${ }^{9}$, Woong Sun ${ }^{2,5}$, Hyunkyung Kim ${ }^{2,10}$, \\ Jin Young Kim ${ }^{7}$, Eunjoon Kim ${ }^{4,6}$ and Kihoon Han ${ }^{1,2 *}$
}

\begin{abstract}
${ }^{1}$ Department of Neuroscience, College of Medicine, Korea University, Seoul, South Korea, ${ }^{2}$ Department of Biomedical Sciences, College of Medicine, Korea University, Seoul, South Korea, ${ }^{3}$ Division of National Supercomputing, Korea Institute of Science and Technology Information, Daejeon, South Korea, ${ }^{4}$ Center for Synaptic Brain Dysfunctions, Institute for Basic Science, Daejeon, South Korea, ${ }^{5}$ Department of Anatomy, College of Medicine, Korea University, Seoul, South Korea, ${ }^{6}$ Department of Biological Sciences, Korea Advanced Institute of Science and Technology, Daejeon, South Korea, ${ }^{7}$ Research Center for Bioconvergence Analysis, Korea Basic Science Institute, Ochang, South Korea, ${ }^{8}$ College of Pharmacy, Korea University, Sejong, South Korea, ${ }^{9}$ Center for Functional Connectomics, Korea Institute of Science and Technology, Brain Science Institute, Seoul, South Korea, ${ }^{10}$ Department of Biochemistry and Molecular Biology, College of Medicine, Korea University, Seoul, South Korea
\end{abstract}

Variants of the SH3 and multiple ankyrin repeat domains 3 (SHANK3), which encodes postsynaptic scaffolds, are associated with brain disorders. The targeted alleles in a few Shank3 knock-out (KO) lines contain a neomycin resistance (Neo) cassette, which may perturb the normal expression of neighboring genes; however, this has not been investigated in detail. We previously reported an unexpected increase in the mRNA expression of Shank3 exons 1-12 in the brains of Shank3B KO mice generated by replacing Shank3 exons 13-16 with the Neo cassette. In this study, we confirmed that the increased Shank3 mRNA in Shank3B KO brains produced an unusual $\sim 60 \mathrm{kDa}$ Shank3 isoform (Shank3-N), which did not properly localize to the synaptic compartment. Functionally, Shank3-N overexpression altered the dendritic spine morphology in cultured neurons. Importantly, Shank3-N expression in Shank3B $\mathrm{KO}$ mice was not a compensatory response to a reduction of full-length Shank3 because expression was still detected in the brain after normalizing the level of full-length Shank3. Moreover, in another Shank3 KO line (Shank3 gKO) with a similar Shank3 exonal deletion as that in Shank3B KO mice but without a Neo cassette, the mRNA expression levels of Shank3 exons 1-12 were lower than those of wild-type mice and Shank3-N was not detected in the brain. In addition, the expression levels of genes neighboring Shank3 on chromosome 15 were altered in the striatum of Shank3B KO but not Shank3 gKO mice. These results suggest that the Neo cassette has potential off-target effects in Shank3B $\mathrm{KO}$ mice.

Keywords: Shank3B knock-out, Neo cassette, gene expression, off-target effect, mouse chromosome 15 


\section{INTRODUCTION}

The $\mathrm{SH} 3$ and multiple ankyrin repeat domains protein 3 (SHANK3) gene encodes core scaffolding proteins that organize the macromolecular protein complex in the postsynaptic density of glutamatergic excitatory synapses (Sheng and Kim, 2000, 2011). Duplications, deletions, and various point mutations of SHANK3 have been identified in individuals with numerous types of brain disorders, including autism spectrum disorders (ASDs), bipolar disorder, intellectual disability, and schizophrenia, suggesting that Shank3 is critical for proper synaptic development and function (Grabrucker et al., 2011; Guilmatre et al., 2014; Monteiro and Feng, 2017; Ey et al., 2020).

To understand the detailed neurobiological mechanisms underlying SHANK3-associated brain disorders, more than 10 different Shank3 knock-out (KO) mouse lines carrying deletions of different Shank 3 exons (of total 22 exons) have been generated to date, and molecular, cellular, and functional changes in their brains have been characterized (Jiang and Ehlers, 2013; Monteiro and Feng, 2017; Eltokhi et al., 2018). Shank3 produces multiple Shank3 protein isoforms resulting from intragenic promoters and alternative splicing (Wang et al., 2014; Choi et al., 2015). Therefore, the combination of Shank3 isoforms can differ among Shank3 KO lines, explaining phenotypic heterogeneity among the KO lines (Monteiro and Feng, 2017).

Moreover, technically, in the targeted alleles of a few Shank3 KO lines, selection markers for gene targeting in embryonic stem cells, such as the neomycin resistance (Neo) cassette, were not removed (Eltokhi et al., 2018). Importantly, the Neo cassette can affect the expression of neighboring genes in the genome (i.e., off-target effects) (Pham et al., 1996; Scacheri et al., 2001; Meier et al., 2010; West et al., 2016). Therefore, it is possible that the expression levels of the remaining Shank3 exons as well as neighboring genes on mouse chromosome 15 are affected by the Neo cassette in these Shank3 KO lines; however, this has not been carefully considered or investigated.

Shank3B KO mice (Peca et al., 2011) in which exons 13-16 of the Shank3 gene are replaced with the Neo cassette are among the most extensively characterized ASD models. We recently identified an unexpected increase in the mRNA expression of Shank3 exons 1-12 (i.e., $5^{\prime}$ of the Neo cassette) in the brains of Shank3B KO mice (Jin et al., 2019a; Lee et al., 2019a). In particular, the mRNA levels of Shank3 exons 1-12 increased significantly in the cortex, striatum, and hippocampus of both juvenile and adult Shank3B KO mice. Furthermore, in multiple brain regions of Shank3B KO mice, the increased mRNA of Shank3 exons 1-12 possibly produced an unusual $\sim 60 \mathrm{kDa}$ Shank3 protein isoform containing the $\mathrm{N}$-terminal domains of Shank3, which was not expressed in wild-type (WT) brains (Jin et al., 2019a). In a previous study, we speculated that the increase in mRNA levels of Shank3 exons 1-12 could be due to the compensatory activation of distal Shank3 promoters in response to reduced Shank 3 protein levels in Shank3B KO brains (Jin et al., 2019a).

In this study, however, we provide several lines of evidence suggesting that the increased mRNA and protein expression levels of Shank3 exons 1-12 in Shank3B KO brains can likely be attributed to off-target effects of the Neo cassette which is retained in the targeted allele. Moreover, we show that the expression levels of genes neighboring Shank 3 on mouse chromosome 15 differ between Shank3B KO brains and WT brains. Together, our results highlight the need for the careful characterization and interpretation of the phenotypes in Shank3B KO mice, and potentially other Shank3 KO lines with the Neo cassette in the targeted allele, especially with respect to gene expression changes.

\section{MATERIALS AND METHODS}

\section{Mice}

The Shank3B KO ( $\Delta 13-16)$, Shank3 global KO (gKO) $(\Delta 14-$ $16)$, and enhanced green fluorescent protein (EGFP)-Shank3 transgenic (TG) mice used in this study have been described previously (Peca et al., 2011; Han et al., 2013b; Lee et al., 2017a; Yoo T. et al., 2018; Yoo T. et al., 2019). The mice were bred and maintained on a C57BL/6J (Japan SLC) background according to the Korea University College of Medicine Research Requirements. All procedures were approved by the Committee on Animal Research at Korea University College of Medicine (KOREA-2018-0003). The mice were fed and had access to water ad libitum and were housed in group (4-6 mice per cage) under a $12 \mathrm{~h}$ light-dark cycle at $18-25^{\circ} \mathrm{C}$. All experiments were performed with adult (8-10 weeks old) male mice, unless otherwise specified.

\section{RNA Purification and Real-Time Quantitative Reverse Transcription PCR (qRT-PCR)}

qRT-PCR was performed as described previously (Jin et al., 2018; Zhang et al., 2018; Lee et al., 2019a). Briefly, total RNA was extracted from tissue samples using an miRNeasy Mini Kit (Qiagen, \#217004) and $2 \mu \mathrm{g}$ of total RNA was used for cDNA synthesis using the iScript cDNA Synthesis Kit (Bio-Rad, \#BR170-8891). Target mRNAs were detected and quantified by a real-time PCR instrument (CFX96 Touch; Bio-Rad) using SYBR Green Master Mix (Bio-Rad, \#BT170-8884AP). The results were analyzed using the comparative $\mathrm{Ct}$ method and were normalized against the levels of housekeeping gene Gapdh (Han et al., 2013a). The primer sequences for real-time PCR were described previously (Jin et al., 2019a) or are as follows:

Mouse Gapdh

Forward 5'-GGCATTGCTCTCAATGACAA-3' Reverse 5'-CCCTGTTGCTGTAGCCGTAT-3'

\section{Mouse Acvrl1}

Forward 5'-GGGCCTTTTGATGCTGTCG- $3^{\prime}$ Reverse 5'-TGGCAGAATGGTCTCTTGCAG-3'

\section{Mouse Adamts20}

Forward 5'-GTCCTGGGAAGTTCGTTTCCA-3' Reverse 5'-GGCTGAAATGCCGGTTCTG-3' 


\author{
Mouse Cers5 \\ Forward $5^{\prime}$-TGCTGTTTGAGCGATTTATTGC-3' \\ Reverse 5'-GGTTCCACCTTATTGACAGGAC-3'
}

Mouse Cpne8

Forward 5'-ACATTGGGGGAGATTGTTGGT-3'

Reverse 5'-ACTTCTGTCTTGTGGCAAATTGT-3'

Mouse Dennd6b

Forward 5'-TTCCGCCCCTACTTCACCAT-3'

Reverse 5'-AGAAAGGGTTTGTGACTCCCA-3'

Mouse Slc11a2

Forward 5'-CAATGTCTTTGTCGTGTCCGT- $3^{\prime}$

Reverse 5'-GCGACCATTTTAGGTTCAGGAAT-3'

\section{Biochemical Analysis and Western Blotting}

Whole lysates and subcellular fractions of mouse tissue samples were prepared as described previously (Han et al., 2009; Jin et al., 2019a,b). Briefly, for whole lysate preparation, frozen mouse tissues were homogenized in RIPA buffer $(50 \mathrm{mM}$ Tris- $\mathrm{HCl} \mathrm{pH}$ 8.0, $150 \mathrm{mM} \mathrm{NaCl}, 0.1 \%$ SDS, $1 \%$ Triton X-100, $0.5 \%$ sodium deoxycholate) with freshly added protease and phosphatase inhibitors (Sigma-Aldrich, \#04906837001 and \#05892970001). The protein concentration was measured using the Bradford Protein Assay (Bio-Rad, \#500-0006). The lysate was heated in $1 \times$ NuPAGE LDS sample buffer (Thermo Fisher Scientific, \#NP0007) containing $1 \times$ NuPAGE reducing agent (Thermo Fisher Scientific, \#NP0004). From each sample, 10-20 mg of protein was loaded onto 4-15\% Mini-PROTEAN TGX Precast Protein Gels (Bio-Rad, \#4561084) for western blotting. The proteins were transferred to the PVDF Membrane (Millipore, \#IPVH00010). Antibodies used for western blot analysis were GAPDH (Cell Signaling, \#2118, 1:3,000), Histone H3 (Cell Signaling, \#4499, 1:2,000), Homer1b/c (Synaptic Systems, 160002, 1:1,000), PSD-95 (Thermo Scientific, MA1-046, 1:1,000), and Shank3 N-terminal (aa 192-221, 1:1,000) (Lee J. et al., 2015). Western blot images were acquired using the ChemiDoc Touch Imaging System (Bio-Rad) and quantified using ImageJ software.

\section{Neuron Culture, Transfection, and Immunocytochemistry}

Cultured hippocampal neurons were prepared from embryonic day 18 rat brains as described previously (Lee et al., 2017c, Lee et al., 2019b; Jin et al., 2018). Dissociated neurons on polyL-lysine-coated coverslips were placed in Neurobasal medium supplemented with B27 (Invitrogen), $0.5 \mathrm{mM} \mathrm{L}$-glutamine, and penicillin/streptomycin (Thermo Fisher Scientific). For immunocytochemistry, cultured hippocampal neurons at days in vitro (DIV) 7 were transfected with EGFP alone (pEGFP-N1) or with EGFP and HA-Shank3-N (pCS2 HA-rat Shank3 1-536) constructs using calcium phosphate. The neurons were fixed at DIV 20 with $4 \% \mathrm{PFA} /$ sucrose, permeabilized with $0.2 \%$ Triton X-100, and incubated with GFP (Abcam, ab13970, 1:1,000) and HA (Santa Cruz, sc-7392, 1:500) primary and dye-conjugated secondary antibodies (Jackson ImmunoResearch). Images were acquired by confocal microscopy (Zeiss, LSM800) and quantified using ImageJ software in a blinded manner.

\section{In-Gel Digestion}

Reduction with dithiothreitol and alkylation with iodoacetamide was performed before each gel piece was treated with trypsin to digest the proteins. Gel pieces were washed with distilled water and $100 \%$ acetonitrile, swollen in digestion buffer containing $50 \mathrm{mM}$ ammonium bicarbonate and $500 \mathrm{ng}$ trypsin, and then incubated at $37^{\circ} \mathrm{C}$ for $16 \mathrm{~h}$. Peptides were recovered by two cycles of extraction with $50 \mathrm{mM}$ ammonium bicarbonate and $90 \%$ acetonitrile. The resulting peptide extracts from each gel piece were lyophilized and stored at $-20^{\circ} \mathrm{C}$ until mass spectrometry (MS) analysis.

\section{Liquid Chromatography (LC)-MS/MS Analysis}

Peptides were analyzed using a LC-MS/MS system consisting of an Ultimate 3000 HPLC (Thermo Fisher scientific) and an Orbitrap Eclipse mass spectrometer (Thermo Fisher Scientific) equipped with a nano-electrospray source (EASY-Spray Sources; Thermo Fisher Scientific). Peptides were trapped on 75 $\mu \mathrm{m} \times 2 \mathrm{~cm}$ C18 precolumn (nanoViper, Acclaim PepMap100; Thermo Fisher Scientific) before being separated on an analytical C18 column $(75 \mu \mathrm{m} \times 50 \mathrm{~cm}$ PepMap RSLC; Thermo Fisher Scientific) at a flow rate of $250 \mathrm{nl} / \mathrm{min}$. The mobile phases A and $\mathrm{B}$ were composed of 0 and $100 \%$ acetonitrile containing $0.1 \%$ formic acid, respectively. The LC gradient began with $5 \% \mathrm{~B}$ and was stayed at $5 \% \mathrm{~B}$ for $10 \mathrm{~min}$, ramped to $13 \% \mathrm{~B}$ for $50 \mathrm{~min}$, to $25 \%$ B for $65 \mathrm{~min}$, to $95 \%$ for $5 \mathrm{~min}$, and remained at $95 \% \mathrm{~B}$ over $5 \mathrm{~min}$. Finally, it was ramped to $5 \% \mathrm{~B}$ for another $1 \mathrm{~min}$. The column was re-equilibrated with $5 \%$ B for 14 min before the next run. The voltage applied to produce an electrospray was 2,050 V. During the chromatographic separation, the Orbitrap Eclipse was operated in data-dependent mode, automatically switching between MS1 and MS2. The MS data were acquired using the following parameters: full scan MS1 spectral (400$2,000 \mathrm{~m} / \mathrm{z}$ ) were acquired in the Orbitrap for a maximum ion injection time of auto mode at a resolution of 120,000 and an automatic gain control (AGC) target of standard mode. MS2 spectra were acquired in the Orbitrap mass analyzer at resolution of 30,000 with high-energy collision dissociation of 27\% normalized collision energy and AGC target of standard mode with maximum ion injection time of dynamic mode. Previously fragmented ions were excluded for $30 \mathrm{~s}$.

\section{Analysis of Mass Spectrometry Data}

MS/MS spectra were analyzed using the following software analysis protocol with the Uniprot mouse database (release at September 18, 2019) including expected amino acid sequence of Shank3-N (1-549 aa). The reversed sequences of all proteins were appended into the database for calculation of false discovery rate (FDR). ProLuCID (Xu et al., 2006) in Integrated Proteomics Pipeline software (IP2) ${ }^{1}$ was used to identify the peptides, a

${ }^{1}$ www.integratedproteomics.com 
precursor mass error of $5 \mathrm{ppm}$, and a fragment ion mass error of $20 \mathrm{ppm}$. Trypsin was selected as a protease, with two potential missed cleavages. Carbamidomethylation at cysteine was chosen as a static modification. Oxidation at methionine was chosen as variable modifications. The output data files were filtered and sorted to compose the protein list with two and more peptides assignments for spectrum identification at a false positive rate less than 0.01. Finally, all identified tandem mass spectra as Shank3-specific peptides were manually validated.

\section{Quantification and Statistical Analysis}

Values from at least three independent experiments with biological replicates were used for quantification and statistical analyses. All analyses were carried out in a blinded manner. $P$ values were calculated by two-tailed Student's $t$-tests or by one-way or two-way ANOVA with Bonferroni post hoc test using GraphPad Prism 5 software. All data are presented as mean \pm SEM. ${ }^{*} P<0.05 ;{ }^{* *} P<0.01 ;{ }^{* * *} P<0.001$.

\section{RESULTS}

We have previously shown that the mRNA expression of Shank3 exons 1-12 is higher in Shank3B KO brains than in WT brains, as determined by Shank3 exon-specific qRT-PCR analyses (Jin et al., 2019a). Moreover, we detected an approximately $60 \mathrm{kDa}$ Shank3 protein isoform in multiple brain regions of Shank3B $\mathrm{KO}$, but not WT, mice, which may be the product of Shank3 exons 1-12 in Shank3B KO brains (Figure 1A). To better determine the identity of the $\sim 60 \mathrm{kDa}$ Shank3 protein expressed in Shank3B KO brains, we expressed an untagged Shank3 protein in HEK293T cells (Shank3-N, aa 1-536 of 1,730 aa full-length Shank3), corresponding to the Shank3 protein residues encoded by Shank3 exons 1-12 (Figure 1B). A side-by-side gel running and western blot analysis showed that the Shank3-N protein expressed in HEK293T cells had a similar molecular weight to that of the short Shank3 isoform expressed specifically in Shank3B KO brains (Figure 1C), further suggesting that the short Shank3 isoform (now referred to as Shank3-N) was indeed the product of Shank3 exons 1-12.

Because Shank3-N did not contain the C-terminal sterile alpha motif (SAM) domain of full-length Shank3 (Figure 1B), which is critical for synaptic targeting and multimerization (Boeckers et al., 2005; Baron et al., 2006; Hayashi et al., 2009), we investigated its subcellular distribution in the striatum, cortex, and hippocampus of Shank3B KO mice. As expected, full-length Shank3 expressed in the brain regions of WT mice were more highly enriched in the synaptosomal fraction (P2) than in the cytosolic fraction (S2) (Figure 1D-G). In contrast, Shank3-N expressed in Shank3B KO brains showed more cytosolic and less synaptosomal distribution than full-length Shank3 in WT mice, suggesting that Shank3-N is not properly targeted to the synaptic compartment. To better determine that the $\sim 60 \mathrm{kDa}$ protein enriched in the S2 fraction of Shank3B KO brains is Shank3$\mathrm{N}$, we performed mass spectrometry-based analyses on WT and Shank3B KO samples (50-75 $\mathrm{kDa}$ proteins of the SDS-PAGE gel) (Figure 1H). The analyses identified four Shank3-specific peptides (all matched within 1-536 aa residues of Shank3) from the Shank3B KO sample (Supplementary Figure S1). In contrast, no Shank3 peptide was identified from the WT sample.

Based on the aforementioned results, we hypothesized that mislocalized Shank3-N possibly has dominant-negative effects on normal Shank3 functions, such as dendritic spine regulation (Roussignol et al., 2005; Durand et al., 2012), because it contains the N-terminal domains of Shank3 which are involved in interactions with several intracellular signaling regulators (Lilja et al., 2017; Hassani Nia and Kreienkamp, 2018; Yoo Y. E. et al., 2019; Cai et al., 2020; Hassani Nia et al., 2020). To examine this, we co-transfected EGFP and HA-tagged Shank3-N constructs in cultured hippocampal neurons at DIV 7 and analyzed dendritic spines at DIV 20. We observed that Shank3-N proteins were expressed diffusely in cultured neurons, consistent with the results from western blot analyses (Supplementary Figure S2). When compared with control neurons expressing EGFP alone, neurons expressing EGFP and Shank3-N showed a normal dendritic spine density and width, but a significantly increased spine length, suggesting that the overexpression of Shank3-N affects dendritic spine morphology in cultured hippocampal neurons (Figure 1I).

We previously speculated that the increase in the mRNA expression of Shank3 exons 1-12 and Shank3-N production could be a compensatory response to reduced Shank3 protein levels in Shank3B KO brains (Jin et al., 2019a). To validate this hypothesis in vivo, we crossed Shank3B heterozygous mice (expressing approximately 50\% lower levels of Shank3 than that in WT mice) with EGFP-Shank3 overexpressing transgenic mice (expressing approximately 50\% higher levels of Shank3 than that in WT mice) (Han et al., 2013b; Lee et al., 2017b,d) to normalize Shank3 protein levels. The expression pattern of EGFP-Shank3 in transgenic mice is similar to that of endogenous Shank3 because the mice were generated using a bacterial artificial chromosome containing mouse Shank3 gene and its upstream promoter region (Han et al., 2013b). Moreover, we have previously shown that crossing the two Shank3 mutant lines not only normalizes Shank3 protein levels but also rescues several behavioral phenotypes, such as locomotor hyperactivity and reduced immobility in the tail-suspension test, observed in EGFP-Shank3 transgenic mice (Han et al., 2013b; Lee et al., 2018). We reasoned that if an increase in the mRNA expression of Shank3 exons 1-12 and the production of Shank3-N in Shank3B mice was indeed due to a reduction in Shank3, the Shank3-N expression at the mRNA and protein levels would disappear by crossing Shank3B heterozygous mice with EGFPShank3 transgenic mice.

Because the interpretation of qRT-PCR results for Shank3 exons 1-12 are complicated in Shank3B; EGFP-Shank3 doublemutant mice owing to masking effect by the expression of the EGFP-Shank3 transgene (Jin et al., 2019a), we instead measured the Shank3-N protein levels by western blot analysis. First, we confirmed that Shank3-N is expressed in the striatum of Shank3B heterozygous mice (at a lower level than that in $\mathrm{KO}$ mice); however, it was not expressed in EGFP-Shank3 transgenic mice (Jin et al., 2019a) (Figure 2A). Then, we analyzed the expression levels of Shank3 proteins (both full-length Shank3 
A

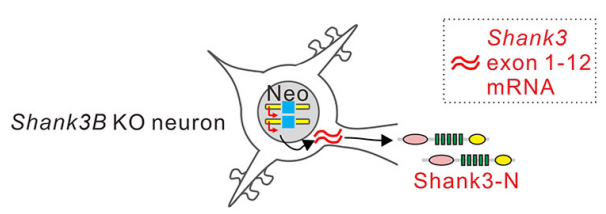

B

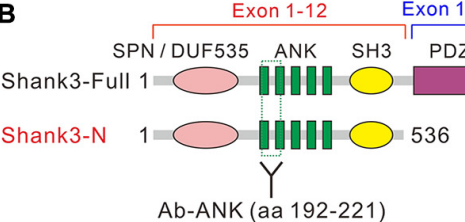

D

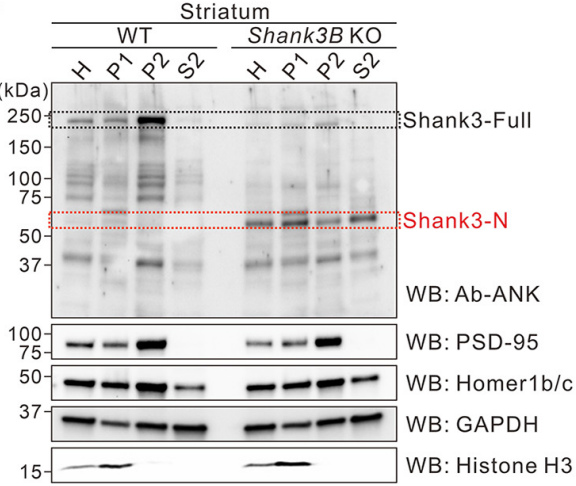

PRO

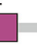

36
$\mathbf{F}$

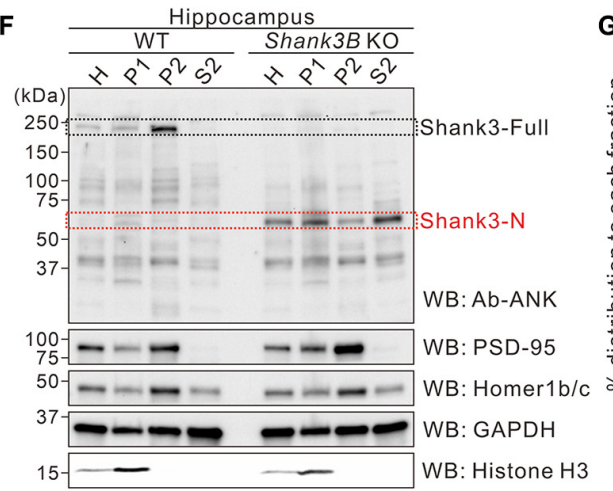

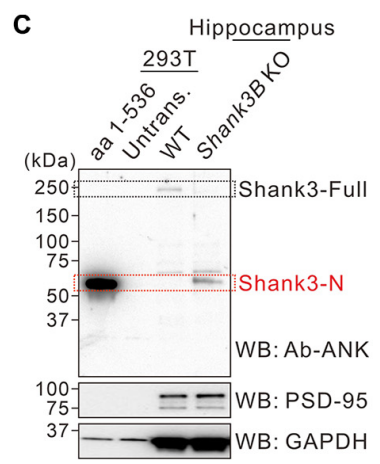

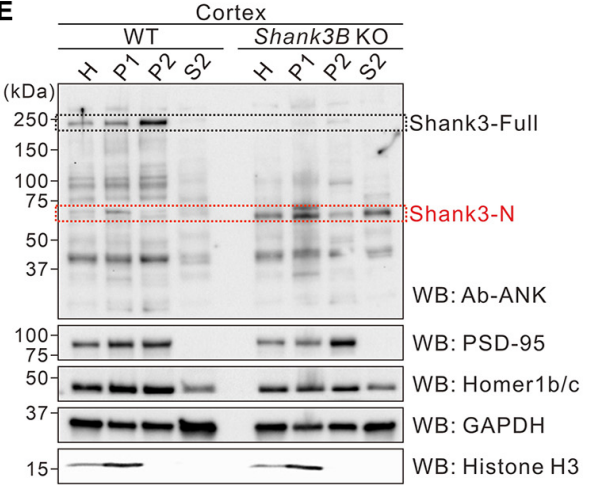

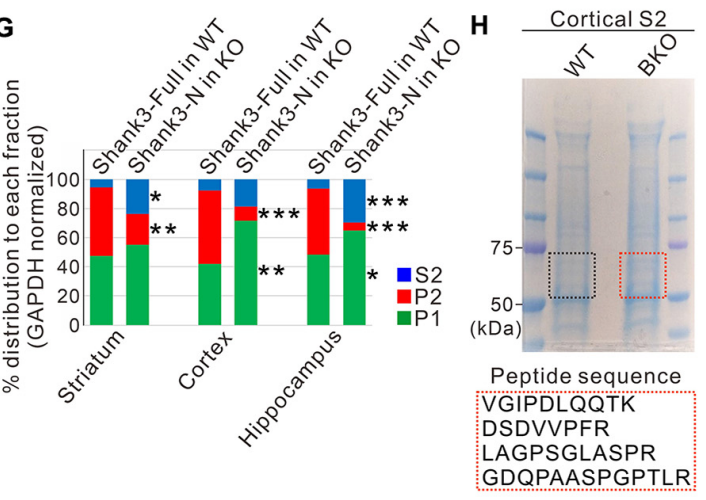
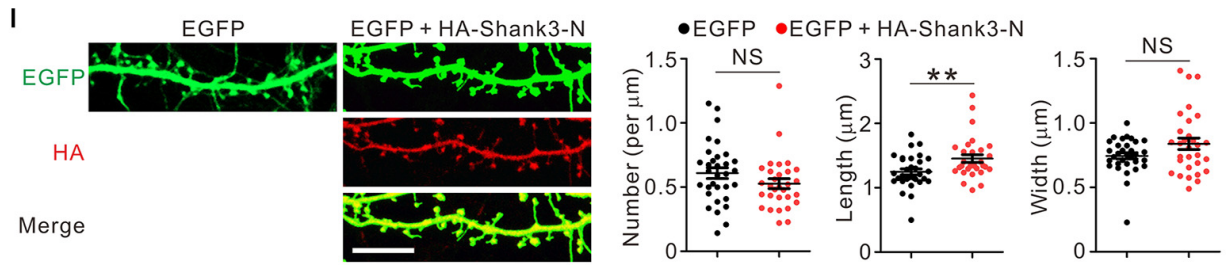

FIGURE 1 | Characterization of the subcellular distribution of Shank3-N protein in Shank3B KO brains. (A) Schematic diagram showing the expression of Shank3 exons 1-12 mRNA and Shank3-N protein in Shank3B knock-out (KO) neurons. Neo, neomycin resistance cassette. (B) Schematic diagram showing the domains of full-length Shank3 (Shank3-Full) and Shank3-N proteins. Location of the epitope for Shank3 N-terminal (Ab-ANK) antibody is indicated. Numbers indicate amino acid residues. ANK, ankyrin repeat domain; DUF535, protein domain of unknown function 535; PDZ, postsynaptic density 95/discs large/zonula occludens 1 domain; PRO, proline-rich region; SAM, sterile alpha motif; SH3, SRC homology 3 domain; SPN, Shank/ProSAP N-terminal domain. (C) Western blot (WB) images showing that Shank3-N protein (aa 1-536) expressed in HEK293T cells and the short Shank3 isoform specifically expressed in the Shank3B KO hippocampus have similar molecular weights. Untrans, untransfected lysate. (D-F) Representative western blot images showing subcellular distributions of Shank3-Full protein in wild-type (WT) brains and Shank3-N protein in Shank3B KO brains. PSD-95 and Homer1b/c are synaptic markers, and Histone H3 is a nuclear marker. H, whole homogenate; P1, pellet \#1 (nuclear fraction); P2, pellet \#2 (crude synaptosomal fraction); S2, supernatant \#2 (cytosolic fraction). (G) Quantification of relative distributions of Shank3-Full and Shank3-N proteins in each subcellular fraction (striatum, two-way ANOVA, genotype $\times$ fraction interaction, $F=7.22, P=0.0087$, Bonferroni post-tests WT vs. KO, $\mathrm{P} 2{ }^{\star *} P<0.01, \mathrm{~S} 2{ }^{*} P<0.05$; cortex, interaction $F=15.43, P=0.0005$, post-tests $\mathrm{P} 1{ }^{* *} P<0.01$, $\mathrm{P} 2{ }^{* \star} P<0.001$; hippocampus interaction $F=29.71, P<0.0001$, posttests $\mathrm{P} 1{ }^{*} P<0.05, \mathrm{P} 2{ }^{* \star *} P<0.001, \mathrm{~S} 2{ }^{* \star *} P<0.0001 ; n=4$ mice per genotype). (H) The S2 fraction samples of WT and Shank3B KO 


\section{FIGURE 1 | Continued}

mice were loaded on SDS-PAGE gel and were stained with Coomassie blue. The gel pieces between 50 and $75 \mathrm{kDa}$ size markers were cut from the WT and KO lanes, and were further processed for mass spectrometry analyses. The sequences of four Shank3-specific peptides identified only from the KO sample are shown. The mass spectrum peaks are shown in Supplementary Figure S1. (I) Representative confocal images of dendritic spines of cultured hippocampal neurons overexpressing EGFP alone or EGFP with HA-Shank3-N. Scale bar, $10 \mu \mathrm{m}$. Quantification of the dendritic spine density, length, and width for each condition ( $n=32$ and 30 neurons for EGFP alone and EGFP + HA-Shank3-N, respectively). NS, not significant. Data are presented as mean \pm SEM. ${ }^{\star} P<0.05$, ${ }^{\star \star} P<0.01$, ${ }^{\star \star \star} P<0.001$.
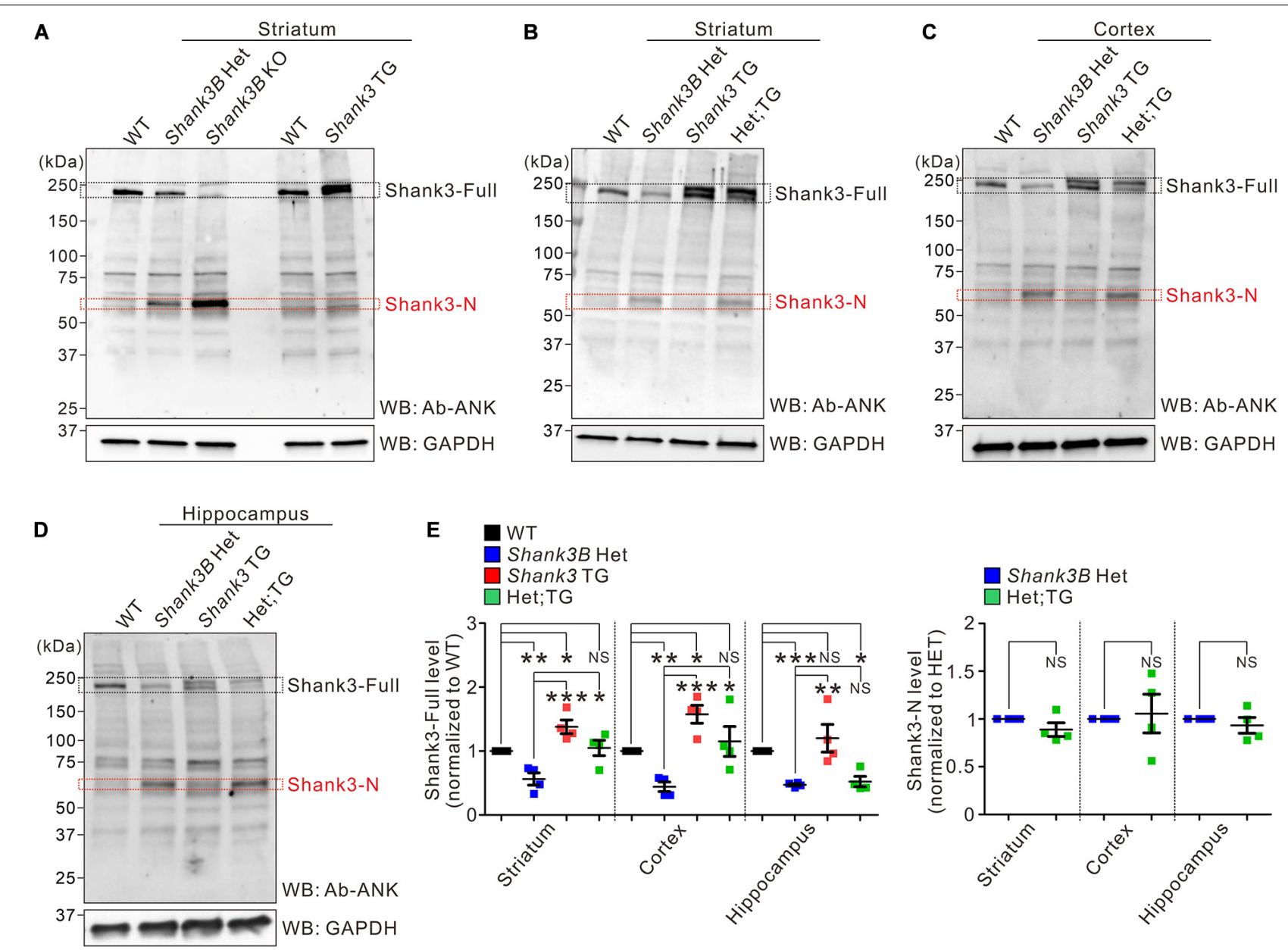

FIGURE 2 | Protein expression of Shank3-N in Shank3B KO brains is not due to a reduction of full-length Shank3 proteins. (A) Western blot (WB) images showing the protein expression of Shank3-N in the striatum of Shank3B heterozygous (HET) and knock-out (KO) mice but not of EGFP-Shank3 overexpressing transgenic (TG) mice. WT, wild-type. (B-D) Representative western blot images showing expression of full-length Shank3 (Shank3-Full) and Shank3-N proteins in the mouse (four different genotypes) striatum, cortex, and hippocampus. Het; TG, double mutant. (E) Quantification of expression levels of Shank3-full (left panel) and Shank3-N (right panel) proteins in the brain regions of mice ( $n=4$ mice per genotype). Note that, in the hippocampus, an increase in Shank3-full expression in Shank3 TG, and thus in Het; TG, mice is milder than those in the striatum and cortex. NS, not significant. Data are presented as mean $\pm \mathrm{SEM} .{ }^{\star} P<0.05,{ }^{\star \star} P<0.01,{ }^{\star \star \star} P<0.001$.

and Shank3-N) in the brain regions (striatum, cortex, and hippocampus) of WT, Shank3B heterozygous, EGFP-Shank3 transgenic, and Shank3B;EGFP-Shank3 double-mutant mice (Figures 2B-D). First, as shown previously (Han et al., 2013b), expression levels of full-length Shank3 in the striatum and cortex of double-mutant mice were significantly higher than those of Shank $3 B$ heterozygous mice and comparable with those of WT mice (Figure 2E, left panel). Surprisingly, however, Shank3$\mathrm{N}$ was still detected in the double-mutant brains at similar levels to those in Shank3B heterozygous brains (Figure 2E, right panel). These results suggest that the expression of Shank3-N in Shank3B mice could not be a compensatory response to a reduction in Shank3.

The aforementioned results prompted us to investigate genomic changes in the targeted allele of Shank $3 B$ KO mice, which were generated by replacing exons 13-16 of the Shank3 gene with the Neo selection cassette (Peca et al., 2011). Several studies have shown that the Neo cassette can affect the expression of neighboring genes in the genome (Pham et al., 1996; Scacheri et al., 2001; Meier et al., 2010; West et al., 2016). Accordingly, 
we investigated another Shank3 KO mouse line in parallel, which has a similar Shank3 exonal deletion as that in Shank3B KO mice but without a remaining selection cassette in the targeted allele. In particular, we focused on Shank3 gKO mice (Yoo T. et al., 2018; Yoo T. et al., 2019), which were generated by deleting exons 14-16 of Shank3.

To identify precise genomic changes in the targeted alleles, we sequenced the genomic region around Shank3 exons 13-16 of the two Shank3 KO lines (Supplementary Information 1). In terms of the inserted sequence in the targeted allele, an approximately 1.9-kbp Neo cassette sequence containing the promoter, Neo, polyA, loxP, and Frt sites was identified in Shank3B KO mice
(Figure 3A, upper panel). In Shank3 gKO mice, only a 130-bp sequence containing Frt and loxP sites was identified (Figure 3A, lower panel). In terms of the deleted sequence from Shank3, exons 13-16 were deleted in the targeted allele of Shank3B KO mice, but the deletions were partial for exons 13 and 16 (Figure 3B, upper panel). In Shank3 gKO mice, entire exons 14-16 and partial sequences of the flanking introns (introns 13 and 16) were deleted in the targeted allele (Figure 3B, lower panel). A side-by-side comparison of the deleted sequences in the two Shank3 KO lines showed that a 904 bp region of Shank3 gene [from intron 13 (partial) to exon 16 (partial)] was deleted in both lines (Figure 3B). Shank3B KO mice had an additional

A Targeted allele

Shank3B KO ( $\triangle 13-16)$ (Peca et al., 2011)

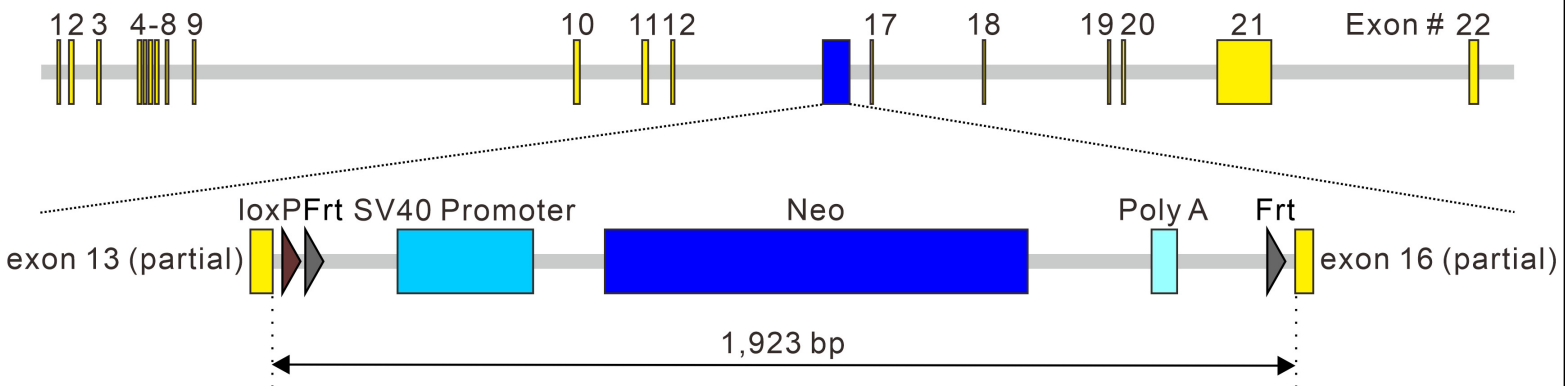

Shank3 gKO ( $\Delta 14-16)$ (Yoo et al., 2018)

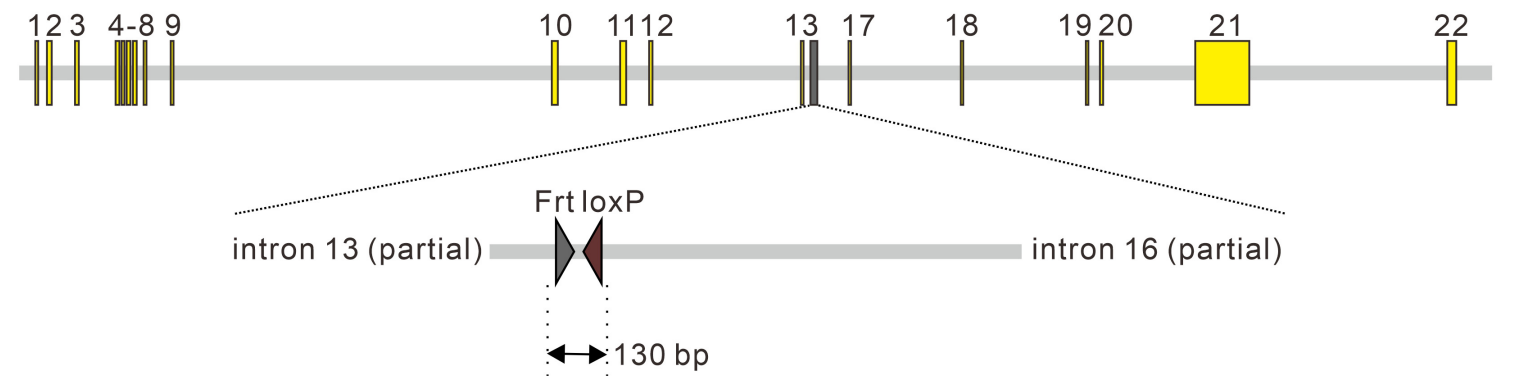

B Deleted region

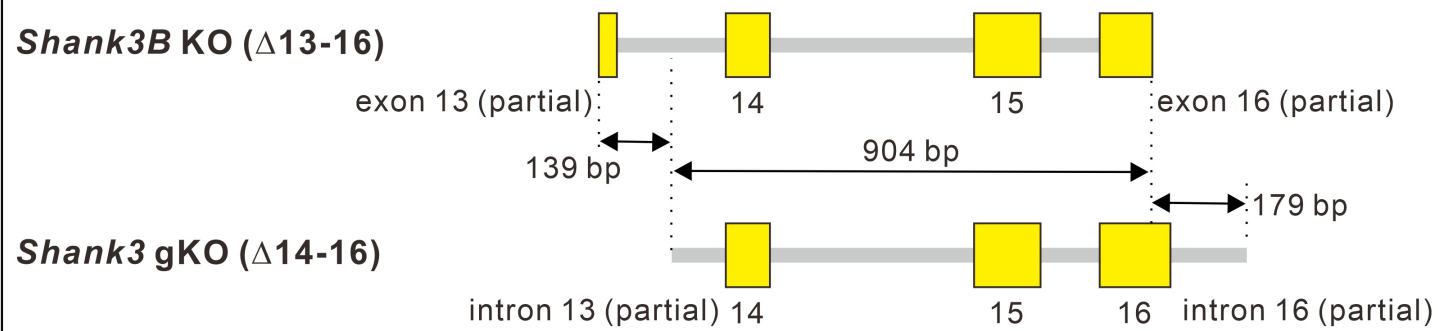

FIGURE 3 | Comparison of the targeted allele sequences of Shank3B KO and Shank3 gKO mice. (A) Schematic diagram showing the inserted sequences of the targeted alleles of Shank3B knock-out (KO) and Shank3 gKO mice. bp, base pair. (B) Schematic diagram showing side-by-side comparison of the deleted Shank3 exon and intron sequences in the targeted alleles of Shank3B KO and Shank3 gKO mice. Full sequencing information is provided in Supplementary Material (Supplementary Information 1). 
139 bp deletion in the $5^{\prime}$ side of Shank3 gene, while Shank3 gKO mice had an additional 179 bp deletion in the $3^{\prime}$ side of Shank3 gene. Taken together, these results suggest that the deleted sequences in the two Shank3 KO lines are largely overlapping, but the inserted sequences in the targeted allele differ substantially (1,923 vs. $130 \mathrm{bp})$.

If the Neo cassette inserted in the targeted allele, rather than a Shank3 protein reduction (as shown in Figure 2), in Shank3B $\mathrm{KO}$ mice is involved in the increase in the mRNA expression of Shank3 exons 1-12, the increase should not be observed in the brain regions of Shank3 gKO mice. First, we confirmed that the mRNA expression levels of Shank3 exons 1-12 increased in the striatum and hippocampus of adult female Shank3B KO mice (Figure 4A), suggesting that this increase occurs in Shank3B $\mathrm{KO}$ mice, regardless of age (at both the juvenile and adult stages) and sex (Jin et al., 2019a). Next, we applied the same Shank3 exon-specific qRT-PCR analysis to the striatum, cortex, hippocampus, and cerebellum of Shank 3 gKO mice. Notably, in all four brain regions of Shank3 gKO mice, mRNA expression levels of all Shank3 exons 1-22 were lower than those in WT mice (Figure 4B). Consistent with the mRNA analysis, even with the loss of full-length Shank3 proteins as in Shank3B KO mice, the $\sim 60 \mathrm{kDa}$ Shank3-N protein was not detected in the Shank3 gKO striatum (Figure 4C). This result further suggests that expression of Shank3-N in Shank3B KO mice was not due to a reduction of full-length Shank3 protein (Figure 2). Taken together, these results demonstrate a significant contrast in mRNA and protein expression patterns of Shank3 exons 1-12 between Shank3B KO and Shank3 gKO brains.

To better understand the Shank3 mRNA and protein products in Shank3B KO and Shank3 gKO brains, we performed PCR with the striatal cDNA of Shank3B KO and Shank3 gKO mice using a forward primer targeting exon 11 and reverse primers targeting different regions of the targeted alleles (Figures 4D,E and Supplementary Information 2). In the case of Shank3B KO mice, PCR products were observed when the forward primer was combined with reverse primers targeting after the SV40 promoter sequence, suggesting that Shank-N mRNA may end with the Neo and polyA sequence of the cassette (Figure 4D, upper panel). Indeed, direct sequencing of the PCR products showed that Shank3 exon 12, skipping the partial exon 13, spliced on the region between SV40 promoter and Neo (Figure 4D, lower panel and Supplementary Information 3). This splicing event may add 13 aa residues (EAEAASASELFQK) to the $\mathrm{C}$ terminus of 536 aa residues encoded by Shank3 exons 1-12 to produce Shank3-N protein (total 549 aa residues). In the case of Shank3 gKO mice, similar PCR and sequencing approaches showed that Shank3 exon 13 spliced on exon 17, which induces frameshift and a premature stop codon (Figure 4E and Supplementary Information 3). We speculate that this Shank3 mRNA in Shank3 gKO brains may undergo nonsense-mediated decay because its mRNA levels were very low (Figure 4B) and because expected proteins from the mRNA were not detected (Figure 4C).

The Neo cassette can have long-range off-target effects on the expression of neighboring genes (Pham et al., 1996). Therefore, we investigated expression changes in genes neighboring Shank3 on mouse chromosome 15 of Shank3B KO mice. Notably, in our previous RNA-sequencing analysis of the striatum of Shank3B KO mice (Lee et al., 2019a), we found that 7 out of 17 (approximately 41\%, including Shank3) differentially expressed genes (DEGs) were on mouse chromosome 15 (Figure 4F). Other than Shank3, three DEGs (Cers5, Adamts20, and Cpne8) were upregulated, and the other three DEGs (Dennd6b, Slc11a2, and Acvrl1) were downregulated in the striatum of Shank3B KO mice. To validate these results, we performed qRT-PCR analyses of the six genes in the striata of both Shank3B KO and Shank3 gKO mice, together with their respective WT littermates. In the Shank3B KO striatum, expression levels of Cers5 were significantly higher and levels of Dennd6b and Acvrl1 were significantly lower than those in the WT striatum (Figure 4G). In contrast, however, none of the six genes showed significant expression changes in Shank3 gKO mice, suggesting that changes in the expression of genes neighboring Shank3 occur specifically in Shank3B KO mice.

To further understand the overall gene expression changes in the Shank3B KO striatum, we expanded the DEG list from 17 to 344 by applying a less stringent statistical criterion (from FDR $<0.05$ to $P<0.01)$. Notably, when normalized to the total number of genes on chromosome 15 (673 genes), 2.8\% of genes (19 out of 673) were identified in the list of 334 DEGs, which was the highest percentage among the 20 mouse chromosomes (average $1.9 \%$ of genes) (Figure $\mathbf{4 H}$ ). Moreover, when the 344 DEGs were ranked based on $P$ values, the average rank value of the 19 DEGs on chromosome 15 was the lowest (i.e., the DEGs were top-ranked) compared with average ranks of DEGs on other chromosomes (Figure $4 \mathbf{H}$, red bars). These results suggest that, in the striatum of Shank3B KO mice, gene expression changes were overrepresented on chromosome 15.

\section{DISCUSSION}

In this study, we showed that Shank3-N protein expressed in Shank3B KO brains was less efficiently targeted to the synaptic compartment than full-length Shank3, possibly because it has only the N-terminal [Shank/ProSAP N-terminal (SPN), ankyrin repeat (ANK), and SRC homology 3 (SH3)] domains but not the C-terminal SAM domain of full-length Shank3. The N-terminal domains of Shank3 interact with several proteins, such as Ras and Rap G-proteins (Lilja et al., 2017), $\alpha$-fordin (Bockers et al., 2001), Densin-180 (Quitsch et al., 2005), $\mathrm{Ca}_{\mathrm{v}} 1.3$ L-type calcium channel (Zhang et al., 2005), and Sharpin (Lim et al., 2001), which are involved in the regulation of diverse neuronal functions. We carefully speculate that Shank3-N protein may possibly interact with some of the aforementioned proteins, and thereby disturbing their proper synaptic localization and function. Moreover, a recent study has identified nuclear localization signals in the N-terminal part of Shank3 (between the ANK and SH3 domains) (Hassani Nia et al., 2020). Supporting its functional importance, several types of missense mutations in the N-terminal part of Shank3 have been identified in patients with ASDs (Hassani Nia and Kreienkamp, 2018). Therefore, Shank3-N expression, together with loss of full-length Shank3, may, at least partially, contribute to the neuronal phenotypes 

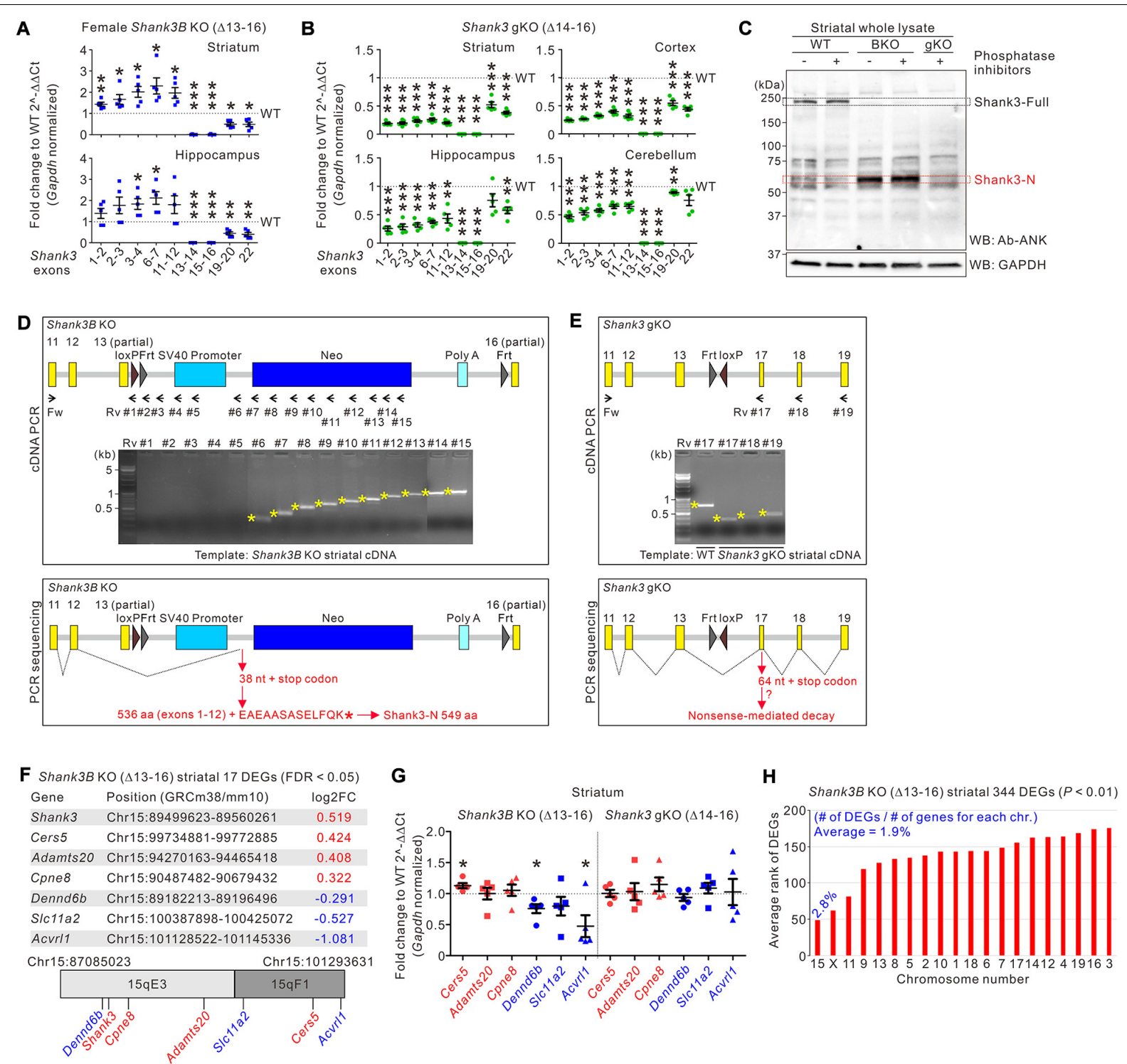

FIGURE 4 | Abnormal expression of Shank3 exons 1-12 and neighboring genes on mouse chromosome 15 in Shank3B KO brains. (A) qRT-PCR analysis of exon-specific Shank3 mRNA levels in the striatum and hippocampus of adult female Shank3B knock-out (KO) mice compared with wild-type $(\mathrm{WT})$ littermates $(n=5$ animals per genotype). (B) qRT-PCR analysis of exon-specific Shank3 mRNA levels in the striatum, cortex, hippocampus, and cerebellum of adult male Shank3 gKO mice compared with WT littermates ( $n=5$ animals per genotype). (C) Western blot (WB) images showing that Shank3-N protein is not expressed in the striatum of Shank3 gKO mice. The molecular weight of Shank3-N protein in the Shank3B KO striatum was not significantly changed by omission of phosphatase inhibitors during protein sample preparation, suggesting that Shank3-N is not highly modified by phosphorylation. (D) Schematic diagram showing the locations of PCR primers targeting the Neo cassette of Shank3B KO mice. Agarose gel image showing the results of PCR reactions with the primer sets (upper panel). Schematic diagram showing the splicing of Shank3 exon 12 on the Neo cassette (lower panel). The splicing adds 13 aa residues to Shank3 encoded by exons 1-12 to produce Shank3-N protein (549 aa residues). Fw, forward; Rv, reverse. (E) Schematic diagrams showing the locations of PCR primers (upper panel) and the splicing of Shank3 exons in Shank3 gKO mice (lower panel). The splicing causes frameshift and generates a premature stop codon, which may lead to nonsense-mediated decay of the mRNA. (F) Summary of the seven differentially expressed genes (DEGs) in the Shank3B KO striatum on mouse chromosome 15. Their genomic positions and fold change (FC) values are indicated. (G) qRT-PCR analysis of the six DEGs on mouse chromosome 15 in the striatum of Shank3B KO and Shank3 gKO mice compared with respective WT controls ( $n=5$ animals per genotype). (H) Chromosomal distribution (normalized to total number of genes for each chromosome) and rank values of 344 DEGs in the Shank3B KO striatum. chr, chromosome. Data are presented as mean \pm SEM. ${ }^{\star} P<0.05$, ${ }^{\star \star} P<0.01$,

${ }^{\star \star \star} P<0.001$

observed in Shank3B KO mice. This is further supported by our observation that Shank3-N overexpression changed dendritic spine morphology in cultured hippocampal neurons. However, this result was obtained in WT neurons expressing endogenous full-length Shank3, and thus may not fully reflect Shank3-N function in Shank3B KO neurons.

We provided several lines of evidence suggesting that the Neo cassette in the targeted allele of Shank3B KO mice has 
potential off-target effects on the expression of Shank3 and its neighboring genes on mouse chromosome 15. First, we excluded the possibility that Shank3-N expression in Shank3B mice was a compensatory response to a reduction of full-length Shank3. Specifically, Shank3-N was still detected even after normalizing the level of full-length Shank3 by crossing Shank3B heterozygous mice with Shank3 overexpressing transgenic mice. In addition, Shank3-N was not detected in the brains of Shank3 gKO mice, despite the loss of full-length Shank3 as in Shank3B KO mice.

Second, we compared the targeted allele sequences of Shank3B KO and Shank3 gKO mice. This revealed that deleted sequences of Shank3 gene in the two KO lines were largely overlapping, while retained sequences of selection markers differed substantially, suggesting that the latter sequences may be more important determinants of gene expression differences between the two KO lines. Moreover, no gene regulatory element was identified around the genomic region of Shank3 exons 1316 (Supplementary Figure S3), suggesting that integration of the exogenous Neo cassette, rather than the removal of the Shank3 sequence, likely resulted in the abnormal gene expression in Shank3B KO brains. By performing PCR with different primer sets followed by direct sequencing, we predicted the Shank3 mRNA and protein products in Shank3B KO and Shank3 gKO mice. We carefully speculate that the Shank3 mRNA in Shank3 gKO, but not Shank3B KO, brains may undergo nonsensemediated decay. It is not immediately clear how Shank3-N mRNA in Shank3B KO mice escapes from nonsense-mediated decay. Nevertheless, the escape alone may not be enough to fully explain the expression of Shank3-N mRNA in Shank3B KO mice because its levels are upregulated compared with Shank3 transcripts in WT mice.

Third, in addition to Shank3 exons 1-12, neighboring genes on mouse chromosome 15, such as Cers5, Dennd6b, and Acvrl1, showed altered expression levels in the striatum of Shank3B KO mice, but not in Shank3 gKO mice. Furthermore, genes with expression changes in the striatum of Shank3B KO mice were disproportionately clustered on chromosome 15 (41\% of DEGs). When we re-analyzed the RNA-sequencing data on the prefrontal cortex of another Shank3 KO line (Shank3 $\triangle \mathrm{C}$ carrying deletion of exon 21) (Qin et al., 2018), only 2 out of 45 DEGs (including Shank3 itself) were on chromosome 15 (Jin et al., 2018), further indicating that gene expression changes in Shank3B KO brains are unusual. Nevertheless, whether these effects were solely due to the Neo cassette in the targeted allele of Shank3B KO mice and how the Neo cassette interacted with Shank3 exons 1-12 and its neighboring genes are not immediately clear. Moreover, it cannot be excluded that Shank3-N protein may secondarily affect gene expression in Shank3B KO brains by modulating intracellular signals. Notably, we found that the Neo cassette of Shank3B KO mice is flanked by Frt sites (Figure $\mathbf{3 A}$ ). Therefore, it will be an interesting topic for future studies to determine whether the Neo cassette can be removed using the Frt sites and whether expression changes of Shank3 exons 1-12 and neighboring genes in Shank3B KO mice can be attenuated by removing the Neo cassette. Moreover, it will be another interesting topic to investigate whether those genes (Cers5, Dennd6b, and Acvrl1) on chromosome 15 with altered mRNA expression levels in
Shank3B KO mice have any functional effects. Validation of their protein level changes (ceramide synthase 5, DENN domain containing $6 \mathrm{~B}$, and activin $\mathrm{A}$ receptor like type 1 which are encoded by Cers5, Dennd6b, and Acvrl1, respectively) in Shank3B KO brains compared with WT brains is a prerequisite for addressing this issue.

In conclusion, our results emphasize the need for the careful characterization and interpretation of gene expression changes in Shank3B KO mice. More broadly, it is possible that our findings can be extended to other Shank3, and Shank1 and Shank2, KO lines (Eltokhi et al., 2018) with selection markers retained in the targeted alleles. Potential off-target effects of selection markers, together with Shank3 isoform diversity (Wang et al., 2014) and compensatory changes in Shank3 expression (Qin et al., 2018; Jin et al., 2019a), may collectively contribute to phenotypic heterogeneity among different Shank3 KO lines, validation of which requires further investigations.

\section{DATA AVAILABILITY STATEMENT}

All raw MS and supporting data files from this study have been deposited to the repository MassIVE with identifier PDX022828, https://massive.ucsd.edu/ProteoSAFe/dataset.jsp? task=ac7adebf965441f298dbf993e9a28058.

\section{ETHICS STATEMENT}

The animal study was reviewed and approved by the Committee on Animal Research at Korea University College of Medicine.

\section{AUTHOR CONTRIBUTIONS}

CJ, TY, JR, Y-EY, RM, YZ, HRK, YK, HS, GB, SP, and KH designed and performed the experiments. HKa, JK, S-KK, WS, $\mathrm{HKi}, \mathrm{EK}$, and $\mathrm{KH}$ analyzed and interpreted the data. $\mathrm{KH}$ wrote the manuscript. All authors read and approved the article.

\section{FUNDING}

This work was supported by the National Research Foundation of Korea (NRF) grants funded by the Korea Government Ministry of Science and ICT (NRF-2018R1C1B6001235, NRF2018M3C7A1024603 to KH), by the Institute for Basic Science (IBS-R002-D1 to EK), by the Korea University Graduate School Junior Fellow Research Grant (to CJ), by the Korea University (K2020391 to KH), and by the Korea Basic Science Institute grant (C060100 to JK).

\section{SUPPLEMENTARY MATERIAL}

The Supplementary Material for this article can be found online at: https://www.frontiersin.org/articles/10.3389/fnmol.2020. 614435/full\#supplementary-material 


\section{REFERENCES}

Baron, M. K., Boeckers, T. M., Vaida, B., Faham, S., Gingery, M., Sawaya, M. R., et al. (2006). An architectural framework that may lie at the core of the postsynaptic density. Science 311, 531-535. doi: 10.1126/science.1118995

Bockers, T. M., Mameza, M. G., Kreutz, M. R., Bockmann, J., Weise, C., Buck, F., et al. (2001). Synaptic scaffolding proteins in rat brain. Ankyrin repeats of the multidomain Shank protein family interact with the cytoskeletal protein alpha-fodrin. J. Biol. Chem. 276, 40104-40112. doi: 10.1074/jbc.m102454200

Boeckers, T. M., Liedtke, T., Spilker, C., Dresbach, T., Bockmann, J., Kreutz, M. R., et al. (2005). C-terminal synaptic targeting elements for postsynaptic density proteins ProSAP1/Shank2 and ProSAP2/Shank3. J. Neurochem. 92, 519-524. doi: 10.1111/j.1471-4159.2004.02910.x

Cai, Q., Hosokawa, T., Zeng, M., Hayashi, Y., and Zhang, M. (2020). Shank3 binds to and stabilizes the active form of Rap1 and HRas GTPases via Its NTD-ANK Tandem with distinct mechanisms. Structure 28, 290-300.e294.

Choi, S. Y., Pang, K., Kim, J. Y., Ryu, J. R., Kang, H., Liu, Z., et al. (2015). Posttranscriptional regulation of SHANK3 expression by microRNAs related to multiple neuropsychiatric disorders. Mol. Brain 8:74.

Durand, C. M., Perroy, J., Loll, F., Perrais, D., Fagni, L., Bourgeron, T., et al. (2012). SHANK3 mutations identified in autism lead to modification of dendritic spine morphology via an actin-dependent mechanism. Mol. Psychiatry 17, 71-84. doi: $10.1038 / \mathrm{mp} .2011 .57$

Eltokhi, A., Rappold, G., and Sprengel, R. (2018). Distinct Phenotypes of Shank2 mouse models reflect neuropsychiatric spectrum disorders of human patients with SHANK2 variants. Front. Mol. Neurosci. 11:240. doi: 10.3389/fnmol.2018. 00240

Ey, E., Bourgeron, T., Boeckers, T. M., Kim, E., and Han, K. (2020). Editorial: shankopathies: shank protein deficiency-induced synaptic diseases. Front. Mol. Neurosci. 13:11. doi: 10.3389/fnmol.2020.00011

Grabrucker, A. M., Schmeisser, M. J., Schoen, M., and Boeckers, T. M. (2011). Postsynaptic ProSAP/Shank scaffolds in the cross-hair of synaptopathies. Trends Cell Biol. 21, 594-603. doi: 10.1016/j.tcb.2011.07.003

Guilmatre, A., Huguet, G., Delorme, R., and Bourgeron, T. (2014). The emerging role of SHANK genes in neuropsychiatric disorders. Dev. Neurobiol. 74, 113122. doi: $10.1002 /$ dneu. 22128

Han, K., Gennarino, V. A., Lee, Y., Pang, K., Hashimoto-Torii, K., Choufani, S., et al. (2013a). Human-specific regulation of MeCP2 levels in fetal brains by microRNA miR-483-5p. Genes Dev. 27, 485-490. doi: 10.1101/gad.207456.112

Han, K., Holder, J. L. Jr., Schaaf, C. P., Lu, H., Chen, H., and Kang, H. (2013b). SHANK3 overexpression causes manic-like behaviour with unique pharmacogenetic properties. Nature 503, 72-77. doi: 10.1038/nature12630

Han, K., Kim, M. H., Seeburg, D., Seo, J., Verpelli, C., Han, S., et al. (2009). Regulated RalBP1 binding to RalA and PSD-95 controls AMPA receptor endocytosis and LTD. PLoS Biol. 7:e1000187. doi: 10.1371/journal.pbio. 1000187

Hassani Nia, F., and Kreienkamp, H. J. (2018). Functional relevance of missense mutations affecting the $\mathrm{N}$-terminal part of shank 3 found in autistic patients. Front. Mol. Neurosci. 11:268. doi: 10.3389/fnmol.2018.00268

Hassani Nia, F., Woike, D., Kloth, K., Kortum, F., and Kreienkamp, H. J. (2020). Truncating mutations in SHANK3 associated with global developmental delay interfere with nuclear beta-catenin signaling. J. Neurochem. 155, 250-263. doi: 10.1111 jnc. 15014

Hayashi, M. K., Tang, C., Verpelli, C., Narayanan, R., Stearns, M. H., Xu, R. M., et al. (2009). The postsynaptic density proteins Homer and Shank form a polymeric network structure. Cell 137, 159-171. doi: 10.1016/j.cell.2009.01.050

Jiang, Y. H., and Ehlers, M. D. (2013). Modeling autism by SHANK gene mutations in mice. Neuron 78, 8-27. doi: 10.1016/j.neuron.2013.03.016

Jin, C., Kang, H., Ryu, J. R., Kim, S., Zhang, Y., Lee, Y., et al. (2018). Integrative brain transcriptome analysis reveals region-specific and broad molecular changes in Shank3-overexpressing mice. Front. Mol. Neurosci. 11:250. doi: 10. 3389/fnmol.2018.00250

Jin, C., Kang, H. R., Kang, H., Zhang, Y., Lee, Y., Kim, Y., et al. (2019a). Unexpected compensatory increase in Shank3 transcripts in Shank3 Knock-out mice having partial deletions of exons. Front. Mol. Neurosci. 12:228. doi: 10.3389/fnmol. 2019.00228

Jin, C., Kim, S., Kang, H., Yun, K. N., Lee, Y., Zhang, Y., et al. (2019b). Shank3 regulates striatal synaptic abundance of Cyld, a deubiquitinase specific for
Lys63-linked polyubiquitin chains. J. Neurochem. 150, 776-786. doi: 10.1111/ jnc. 14796

Lee, B., Zhang, Y., Kim, Y., Kim, S., Lee, Y., and Han, K. (2017a). Age-dependent decrease of GAD65/67 mRNAs but normal densities of GABAergic interneurons in the brain regions of Shank3-overexpressing manic mouse model. Neurosci. Lett. 649, 48-54. doi: 10.1016/j.neulet.2017. 04.016

Lee, Y., Kang, H., Lee, B., Zhang, Y., Kim, Y., Kim, S., et al. (2017b). Integrative analysis of brain region-specific Shank3 interactomes for understanding the heterogeneity of neuronal pathophysiology related to SHANK3 mutations. Front. Mol. Neurosci. 10:110. doi: 10.3389/fnmol.2017.00110

Lee, Y., Kim, D., Ryu, J. R., Zhang, Y., Kim, S., Kim, Y., et al. (2017c). Phosphorylation of CYFIP2, a component of the WAVE-regulatory complex, regulates dendritic spine density and neurite outgrowth in cultured hippocampal neurons potentially by affecting the complex assembly. Neuroreport 28, 749-754. doi: 10.1097/wnr.0000000000000838

Lee, Y., Kim, S. G., Lee, B., Zhang, Y., Kim, Y., Kim, S., et al. (2017d). Striatal transcriptome and interactome analysis of Shank3-overexpressing mice reveals the connectivity between Shank3 and mTORC1 signaling. Front. Mol. Neurosci. 10:201. doi: $10.3389 /$ fnmol.2017.00201

Lee, J., Chung, C., Ha, S., Lee, D., Kim, D. Y., Kim, H., et al. (2015). Shank3-mutant mice lacking Exon 9 show altered excitation/inhibition balance, enhanced rearing, and spatial memory deficit. Front. Cell. Neurosci. 9:94. doi: 10.3389/ fncel.2015.00094

Lee, Y., Kang, H., Jin, C., Zhang, Y., Kim, Y., and Han, K. (2019a). Transcriptome analyses suggest minimal effects of Shank3 dosage on directional gene expression changes in the mouse striatum. Anim. Cells Syst. 23, 270-274. doi: 10.1080/19768354.2019.1595142

Lee, Y., Zhang, Y., Ryu, J. R., Kang, H. R., Kim, D., Jin, C., et al. (2019b). Reduced CYFIP2 stability by Arg87 variants causing human neurological disorders. Ann. Neurol. 86, 803-805. doi: 10.1002/ana.25598

Lee, Y., Zhang, Y., Kim, S., and Han, K. (2018). Excitatory and inhibitory synaptic dysfunction in mania: an emerging hypothesis from animal model studies. Exper. Mol. Med. 50:12.

Lilja, J., Zacharchenko, T., Georgiadou, M., Jacquemet, G., De Franceschi, N., Peuhu, E., et al. (2017). SHANK proteins limit integrin activation by directly interacting with Rap1 and R-Ras. Nat. Cell Biol. 19, 292-305. doi: 10.1038/ ncb3487

Lim, S., Sala, C., Yoon, J., Park, S., Kuroda, S., Sheng, M., et al. (2001). Sharpin, a novel postsynaptic density protein that directly interacts with the shank family of proteins. Mol. Cell. Neurosci. 17, 385-397. doi: 10.1006/mcne.2000.0940

Meier, I. D., Bernreuther, C., Tilling, T., Neidhardt, J., Wong, Y. W., Schulze, C., et al. (2010). Short DNA sequences inserted for gene targeting can accidentally interfere with off-target gene expression. FASEB J. 24, 1714-1724. doi: 10.1096/ fi.09-140749

Monteiro, P., and Feng, G. (2017). SHANK proteins: roles at the synapse and in autism spectrum disorder. Nat. Rev. Neurosci. 18, 147-157. doi: 10.1038/nrn. 2016.183

Peca, J., Feliciano, C., Ting, J. T., Wang, W., Wells, M. F., Venkatraman, T. N., et al. (2011). Shank3 mutant mice display autistic-like behaviours and striatal dysfunction. Nature 472, 437-442. doi: 10.1038/nature09965

Pham, C. T., Macivor, D. M., Hug, B. A., Heusel, J. W., and Ley, T. J. (1996). Longrange disruption of gene expression by a selectable marker cassette. Proc. Natl. Acad. Sci. U.S.A. 93, 13090-13095. doi: 10.1073/pnas.93.23.13090

Qin, L., Ma, K., Wang, Z. J., Hu, Z., Matas, E., Wei, J., et al. (2018). Social deficits in Shank3-deficient mouse models of autism are rescued by histone deacetylase (HDAC) inhibition. Nat. Neurosci. 21, 564-575. doi: 10.1038/s41593-0180110-8

Quitsch, A., Berhorster, K., Liew, C. W., Richter, D., and Kreienkamp, H. J. (2005). Postsynaptic shank antagonizes dendrite branching induced by the leucine-rich repeat protein Densin-180. J. Neurosci. 25, 479-487. doi: 10.1523/jneurosci. 2699-04.2005

Roussignol, G., Ango, F., Romorini, S., Tu, J. C., Sala, C., Worley, P. F., et al. (2005). Shank expression is sufficient to induce functional dendritic spine synapses in aspiny neurons. J. Neurosci. 25, 3560-3570. doi: 10.1523/jneurosci.4354-04. 2005

Scacheri, P. C., Crabtree, J. S., Novotny, E. A., Garrett-Beal, L., Chen, A., Edgemon, K. A., et al. (2001). Bidirectional transcriptional activity of PGK-neomycin 
and unexpected embryonic lethality in heterozygote chimeric knockout mice. Genesis 30, 259-263. doi: 10.1002/gene.1072

Sheng, M., and Kim, E. (2000). The Shank family of scaffold proteins. J. Cell Sci. 113(Pt 11), 1851-1856.

Sheng, M., and Kim, E. (2011). The postsynaptic organization of synapses. Cold Spring Harb. Perspect. Biol. 3:a005678. doi: 10.1101/cshperspect.a005678

Wang, X., Xu, Q., Bey, A. L., Lee, Y., and Jiang, Y. H. (2014). Transcriptional and functional complexity of Shank3 provides a molecular framework to understand the phenotypic heterogeneity of SHANK3 causing autism and Shank3 mutant mice. Mol. Autism 5:30. doi: 10.1186/2040-2392-5-30

West, D. B., Engelhard, E. K., Adkisson, M., Nava, A. J., Kirov, J. V., Cipollone, A., et al. (2016). Transcriptome analysis of targeted mouse mutations reveals the topography of local changes in gene expression. PLoS Genet. 12:e1005691. doi: 10.1371/journal.pgen.1005691

Xu, T., Venable, J. D., Park, S. K., Cociorva, D., Lu, B., Liao, L., et al. (2006). ProLuCID, a fast and sensitive tandem mass spectra-based protein identification program. Mol. Cell. Proteom. 5, S174-S174.

Yoo, T., Cho, H., Lee, J., Park, H., Yoo, Y. E., Yang, E., et al. (2018). GABA neuronal deletion of Shank3 Exons 14-16 in mice suppresses striatal excitatory synaptic input and induces social and locomotor abnormalities. Front. Cell Neurosci. 12:341. doi: 10.3389/fncel.2018.00341

Yoo, T., Cho, H., Park, H., Lee, J., and Kim, E. (2019). Shank3 Exons 14-16 deletion in glutamatergic neurons leads to social and repetitive behavioral deficits associated with increased cortical Layer 2/3 Neuronal excitability. Front. Cell Neurosci. 13:458. doi: 10.3389/fncel.2019.00458
Yoo, Y. E., Yoo, T., Lee, S., Lee, J., Kim, D., Han, H. M., et al. (2019). Shank3 mice carrying the human Q321R mutation display enhanced self-grooming, abnormal electroencephalogram patterns, and suppressed neuronal excitability and seizure susceptibility. Front. Mol. Neurosci. 12:155. doi: 10.3389/fnmol. 2019.00155

Zhang, H., Maximov, A., Fu, Y., Xu, F., Tang, T. S., Tkatch, T., et al. (2005). Association of CaV1.3 L-type calcium channels with Shank. J. Neurosci. 25, 1037-1049. doi: 10.1523/jneurosci.4554-04.2005

Zhang, Y., Kang, H., Lee, Y., Kim, Y., Lee, B., Kim, J. Y., et al. (2018). Smaller body size, early postnatal lethality, and cortical extracellular matrix-related gene expression changes of Cyfip2-null embryonic mice. Front. Mol. Neurosci. 11:482. doi: 10.3389/fnmol.2018.00482

Conflict of Interest: The authors declare that the research was conducted in the absence of any commercial or financial relationships that could be construed as a potential conflict of interest.

Copyright (c) 2021 Jin, Kang, Yoo, Ryu, Yoo, Ma, Zhang, Kang, Kim, Seong, Bang, Park, Kwon, Sun, Kim, Kim, Kim and Han. This is an open-access article distributed under the terms of the Creative Commons Attribution License (CC BY). The use, distribution or reproduction in other forums is permitted, provided the original author(s) and the copyright owner(s) are credited and that the original publication in this journal is cited, in accordance with accepted academic practice. No use, distribution or reproduction is permitted which does not comply with these terms. 